\title{
SINGULAR RELATIONS BETWEEN CERTAIN ARITHMETICAL FUNCTIONS*
}

BY

E. T. BELL

\section{INTRODUCTION AND SUMMARIES}

1. Introduction. We shall say that $f(x)$ is an arithmetical function of $x$ if $f(x)$ is uniform and finite for all finite integer values $>0$ of $x$. The arithmetical functions $f(x), g(x)$ are said to be distinct if $f(x) \neq g(x)$ for at least one integer $x>0$.

Let $a_{1}, \cdots, a_{r}$ be numerical constants all different from zero, and let $f_{1}(x), \cdots, f_{r}(x)$ be $r$ distinct arithmetical functions of $x$. Then if and only if

$$
a_{1} f_{1}(x)+\cdots+a_{r} f_{r}(x)=0
$$

has only a finite number of integer solutions $x>0$, we shall call (1.1) a singular relation between $f_{1}(x), \cdots, f_{r}(x)$. In (1.1) precisely one of $f_{1}(x), \cdots, f_{r}(x)$ may be the arithmetical function whose value is 1 for all integers $x>0$. Having obtained a singular relation in any instance we shall require also the statement of all integers $x>0$ which satisfy it. The number of completely solved singular relations known is very small, probably not more than a dozen. In this paper, we add about 50 more, concerning the functions next defined.

The following notation will be used throughout the paper without further reference.

$n, m, p$ denote integers $>0 ; n$ is arbitrary, $m$ odd; and $p$ is an odd prime; 1 is considered as being composite.

$\sigma(n)=$ the sum of the divisors of $n . \dagger$

$\tau(n)=$ the excess of the number of divisors of $n$ of the forms $8 t+1,8 t+3$ over the number of divisors of the forms $8 t+5,8 t+7$.

$\omega(n)=$ the excess of the number of divisors of $n$ of the form $3 t+1$ over the number of divisors of the form $3 t+2$.

$\xi(n)=$ the excess of the number of divisors of $n$ of the form $4 t+1$ over the number of divisors of the form $4 t+3$.

\footnotetext{
* Presented to the Society, November 29, 1930; received by the editors in August, 1930.

$\dagger$ The rudimentary state of the subject is seen from the following. Let $\phi(n)=n$ for all integers $n>0$. The relation $\sigma(n)-2 \phi(n)=0$ is as simple in appearance as any in this paper, yet it is not known whether it is singular. It is not known whether $\sigma(m)-2 \phi(m)=0$ is solvable, although $\sigma(2 n)-4 \phi(n)$ $=0$ is.
} 
$\epsilon(x)=1$ if $x$ is the square of an integer $>0$, and $\epsilon(x)=0$ otherwise.

$12 E(n)=$ the number of representations of $n$ as a sum of 3 squares; $E(n)$ is the usual notation for a class number function.

$F(n)=$ the number of odd classes of binary quadratic (Gauss) forms of determinant $-n$, with all the usual conventions (as in H. J. S. Smith's Report, and similarly for $E(n))$. For example, $F(1)=1 / 2, F(2)=1, \cdots$, $F(5)=2, \cdots, F(9)=5 / 2, \cdots, F(25)=5 / 2, \cdots, F(100)=5$. There is a table of $F(n), n=1, \cdots, 100$, useful in numerical verifications, in the Tôhoku Mathematical Journal, vol. 19 (1921), p. 116.

Writing $f=f\left(x_{1}, \cdots, x_{s}\right)=a_{1} x_{1}^{2}+\cdots+a_{s} x_{s}^{2}$, where $a_{1}, \cdots, a_{s}$ are integers $>0$, we shall denote by $N(n=f)$ the total number of representations of $n$ in $f$ (the roots of the squares $x_{1}^{2}, \cdots, x_{s}^{2}$ being $\left.\vdots 0\right)$; by $R(n=f$ ) the number of representations of $n$ in $f$ in which $x_{1}, \cdots, x_{s}$ are restricted to be $\geqq 0$; and by $R^{\prime}(n=f)$ the number of representations of $n$ in $f$ in which $x_{1}, \cdots, x_{s}$ are restricted to be all distinct and $\geqq 0$.

In a paper which will be published elsewhere, Dr. Gordon Pall has determined for all $s \geqq 3$ the explicit solutions $n$ of

$$
R^{\prime}\left(n=x_{1}^{2}+\cdots+x_{8}^{2}\right)=0 .
$$

We shall assume his results for $s=4$.

(1.3) Theorem. All $n$ for which (1.2) is solvable with $s=4$ are the Pall numbers $2^{2 h} a, h \geqq 0$, where

$a=2,6,10,18,22,34,58,82 ; 1,3,5,7,9,11,13,15,17,19,23,25,27,31,33$, $37,43,47,55,67,73,97,103$.

These then are the only $n$ which are not sums of 4 unequal squares $\geqq 0$.

From (1.3) we shall deduce all the singular relations which it implies. These relations concern the functions $\sigma, \tau, \omega, \xi, \epsilon, F$ above defined.

2. Summary of lemmas. This section states a number of lemmas.

(2.1) Lemma. $2^{8} R\left(n=a_{1} x_{1}^{2}+a_{2} x_{2}^{2}+\cdots+a_{s} x_{8}^{2}\right)=\sum_{i} N\left(n=a_{i} x_{i}^{2}\right)+\sum_{i, j} N$ $\left(n=a_{i} x_{i}^{2}+a_{j} x_{j}^{2}\right)+\cdots+N\left(n=a_{1} x_{1}^{2}+\cdots+a_{s} x_{s}^{2}\right)$, the notation being as in $\$ 1$, and $\sum_{i, j, \ldots, k}$ referring to all choices of $t$ distinct indices $i, j, \cdots, k$, where $t$ is the number of indices, chosen from $1,2, \cdots, s ; t=1,2, \cdots, s$.

This is proved in $\$ 5$.

(2.2) Lemmas. $16 R\left(n=x^{2}+y^{2}+z^{2}+w^{2}\right)=4 N\left(n=x^{2}\right)+6 N\left(n=x^{2}+y^{2}\right)+4 N(n$ $\left.=x^{2}+y^{2}+z^{2}\right)+N\left(n=x^{2}+y^{2}+z^{2}+w^{2}\right)$.

This is an immediate consequence of (2.1), as also are the next. 


$$
\begin{aligned}
8 R\left(n=x^{2}+y^{2}+2 z^{2}\right)= & 2 N\left(n=x^{2}\right)+N\left(n=2 x^{2}\right)+N\left(n=x^{2}+y^{2}\right) \\
& \quad+2 N\left(n=x^{2}+2 y^{2}\right)+N\left(n=x^{2}+y^{2}+2 z^{2}\right) ; \\
4 R\left(n=x^{2}+3 y^{2}\right)= & N\left(n=x^{2}\right)+N\left(n=3 x^{2}\right)+N\left(n=x^{2}+3 y^{2}\right) ; \\
4 R\left(n=2 x^{2}+2 y^{2}\right)= & 2 N\left(n=2 x^{2}\right)+N\left(n=2 x^{2}+2 y^{2}\right) ; \\
2 R\left(n=4 x^{2}\right)= & N\left(n=4 x^{2}\right) .
\end{aligned}
$$

(2.3) Lemma. $R\left(n=x^{2}+y^{2}+z^{2}+w^{2}\right)=R^{\prime}\left(n=x^{2}+y^{2}+z^{2}+w^{2}\right)+6 R^{\prime}\left(n=x^{2}\right.$ $\left.+y^{2}+2 z^{2}\right)+3 R^{\prime}\left(n=2 x^{2}+2 y^{2}\right)+4 R^{\prime}\left(n=x^{2}+3 y^{2}\right)+R^{\prime}\left(n=4 x^{2}\right)$.

For proof, see $\$ 6$.

Directly from the definitions of $R, R^{\prime}$, we have

(2.4) Lemmas. $R^{\prime}\left(n=4 x^{2}\right)=R\left(n=4 x^{2}\right)$;

$$
\begin{aligned}
R^{\prime}\left(n=x^{2}+3 y^{2}\right)= & R\left(n=x^{2}+3 y^{2}\right)-R\left(n=4 x^{2}\right) ; \\
R^{\prime}\left(n=2 x^{2}+2 y^{2}\right)= & R\left(n=2 x^{2}+2 y^{2}\right)-R\left(n=4 x^{2}\right) ; \\
R^{\prime}\left(n=x^{2}+y^{2}+2 z^{2}\right)=R\left(N=x^{2}+y^{2}+2 z^{2}\right)-2 R\left(n=x^{2}+3 y^{2}\right) & -R\left(n=2 x^{2}+2 y^{2}\right)+2 R\left(n=4 x^{2}\right) .
\end{aligned}
$$

In the last of these the preceding results were used to reduce the identity given at once by the definitions,

$$
\begin{aligned}
R\left(n=x^{2}+y^{2}+2 z^{2}\right)= & R^{\prime}\left(n=x^{2}+y^{2}+2 z^{2}\right)+2 R^{\prime}\left(n=x^{2}+3 y^{2}\right) \\
& +R^{\prime}\left(n=2 x^{2}+2 y^{2}\right)+R^{\prime}\left(n=4 x^{2}\right) .
\end{aligned}
$$

Combining (2.2)-(2.4) in an obvious way we reach

(2.5) LemMa. $16 R^{\prime}\left(n=x^{2}+y^{2}+z^{2}+w^{2}\right)=12 N\left(n=x^{2}\right)+12 N\left(n=2 x^{2}\right)+32 N(n$ $\left.=3 x^{2}\right)-48 N\left(n=4 x^{2}\right)-6 N\left(n=x^{2}+y^{2}\right)-24 N\left(n=x^{2}+2 y^{2}\right)+32 N\left(n=x^{2}+3 y^{2}\right)$ $+12 N\left(n=2 x^{2}+2 y^{2}\right)+4 N\left(n=x^{2}+y^{2}+z^{2}\right)-12 N\left(n=x^{2}+y^{2}+2 z^{2}\right)+N\left(n=x^{2}\right.$ $\left.+y^{2}+z^{2}+w^{2}\right)$.

For easy reference we collect some well known results in the next two.

(2.6) Lemmas. If $h$ is an integer $\geqq 0$, and $n=2^{h} m$, then

$N\left(n=x^{2}+y^{2}\right)=4 \xi(m), N\left(n=x^{2}+2 y^{2}\right)=2 \tau(m), N\left(n=x^{2}+3 y^{2}\right)=C \omega(m)$,

where $C=2,0$, or 6 according as $h=0, h$ is odd, or $h$ is even and $>0 ; N\left(n=x^{2}\right.$ $\left.+y^{2}+z^{2}+w^{2}\right)=b \sigma(m), b=8$, or 24 according as $h=0$ or $h>0$.

(2.7) Lemmas. $N\left(n=x^{2}+y^{2}+z^{2}\right)=12 E(n)$;

$$
N\left(m=x^{2}+y^{2}+2 z^{2}\right)=4 F(2 m), N\left(2 n=x^{2}+y^{2}+2 z^{2}\right)=12 E(n) .
$$


Proofs for the last two are given in the American Mathematical Monthly, vol. 31 (1924), p. 128. The following well known reduction formulas will be found useful, $t \geqq 0$ :

$$
\begin{aligned}
& 3 E(8 t+3)=2 F(8 t+3), E(8 t+7)=0, \quad E(4 n)=E(n), \\
& E(4 t+1)=F(4 t+1), \quad E(4 t+2)=F(4 t+2), \quad F(4 n)=2 F(n) .
\end{aligned}
$$

Further reductions, such as $\xi(4 t+3)=0, \epsilon\left(2^{2 t} m\right)=\epsilon(m)$, etc., which are obvious, will be used in stating final forms of theorems without reference.

From (1.3), (2.5) we have the next two.

(2.8) Lemma. If and only if $2 n$ is a Pall number (as in (1.3)),

$$
\begin{array}{rl}
12 & N\left(2 n=x^{2}+y^{2}+2 z^{2}\right)-4 N\left(2 n=x^{2}+y^{2}+z^{2}\right) \\
= & 12 N\left(2 n=x^{2}\right)+12 N\left(n=x^{2}\right)+32 N\left(2 n=3 x^{2}\right)-48 N\left(n=2 x^{2}\right) \\
& -6 N\left(2 n=x^{2}+y^{2}\right)-24 N\left(2 n=x^{2}+2 y^{2}\right)+32 N\left(2 n=x^{2}+3 y^{2}\right) \\
& +12 N\left(n=x^{2}+y^{2}\right)+N\left(2 n=x^{2}+y^{2}+z^{2}+w^{2}\right) .
\end{array}
$$

(2.9) Lемма. If and only if $m$ is one of the odd numbers in (1.3),

$$
\begin{aligned}
& 12 N\left(m=x^{2}+y^{2}+2 z^{2}\right)-4 N\left(m=x^{2}+y^{2}+z^{2}\right) \\
& \quad=12 N\left(m=x^{2}\right)+32 N\left(m=3 y^{2}\right)-6 N\left(m=x^{2}+y^{2}\right) \\
& -24 N\left(m=x^{2}+2 y^{2}\right)+32 N\left(m=x^{2}+3 y^{2}\right)+N\left(m=x^{2}+y^{2}+z^{2}+w^{2}\right) .
\end{aligned}
$$

3. Singular relations. From (2.6), (2.7), (2.9) we have the following.

(3.1) Theorem. The only $m \equiv 7 \bmod 8$ for which

$$
6 F(2 m)=8 \omega(m)+\sigma(m)
$$

are $m=7,15,23,31,47,55,103$.

(3.2) TheOREM. The only $m \equiv 3 \bmod 8$ for which

$$
6 F(2 m)-4 F(m)=8 \epsilon(m / 3)-6 \tau(m)+8 \omega(m)+\sigma(m)
$$

are $m=3,11,19,27,43,67$.

(3.3) Theorem. The only $m \equiv 1 \bmod 8$ for which

$$
6 F(2 m)-6 F(m)=3 \epsilon(m)-3 \xi(m)-6 \tau(m)+8 \omega(m)+\sigma(m)
$$

are $m=1,9,17,25,33,73,97$.

(3.4) Theorem. The only $m \equiv 5 \bmod 8$ for which

$$
6 F(2 m)-6 F(m)=-3 \xi(m)+8 \omega(m)+\sigma(m)
$$

are $m=5,13,37$. 
(3.5) THEOREM. (3.1)-(3.4) contain all the singular relations implied by the odd solutions of (1.2) with $s=4$.

The next are obtained similarly from (2.8) with $n$ therein of the form $2^{2 h} m(h \geqq 0)$. Several simple reductions by $(2.6),(2.7)$, which need not be preserved, have been used.

(3.6) Theorem. There is no $m \equiv 7 \bmod 8$ such that

$$
2 F(2 m)=2 \tau(m)-\sigma(m) .
$$

(3.7) Theorem. The only $m \equiv 3 \bmod 8$ such that

$$
4 F(m)-2 F(2 m)=\sigma(m)-2 \tau(m)
$$

are $m=3,11$.

(3.8) TheOREM. The only $m \equiv 1 \bmod 4$ such that

$$
6 F(m)-2 F(2 m)=\epsilon(m)+\xi(m)+\sigma(m)-2 \tau(m)
$$

are $m=1,5,9,17,29,41$.

The following come in the same way from (2.8) with $n$ therein of the form $2^{h+1} m(h \geqq 0)$.

(3.9) Theorem. The only $m \equiv 7 \bmod 8$ for which

$$
6 F(2 m)=8 \omega(m)-2 \tau(m)+\sigma(m)
$$

are $m=7,15,23,31,47,55,103$.

(3.10) TheOREM. The only $m \equiv 3 \bmod 8$ for which

$$
18 F(2 m)-4 F(m)=8 \epsilon(m / 3)-6 \tau(m)+24 \omega(m)+3 \sigma(m)
$$

are $m=3,11,19,27,43,67$.

(3.11) TheOREM. The only $m \equiv 1 \bmod 4$ for which

$$
6 F(2 m)-2 F(m)=-3 \epsilon(m)+\xi(m)-2 \tau(m)+8 \omega(m)+\sigma(m)
$$

are $m=1,9,13,17,25,33,37,73,97$.

(3.12) THEOREM. (3.6)-(3.11) contain all the singular relations implied by the even solutions of (1.2) with $s=4$.

Hence all singular relations implied by (1.2) with $s=4$ have been obtained.

4. Singular relations with prime argument $p$. In $\S 3$, taking $m=p$ ( $p$ prime, as always), and reducing the results by the definitions of the functions involved, we get the following, which are numbered correspondingly to $\S 3$. 
(4.1) Theorem. The only $p \equiv 7 \bmod 24$ for which $6 F(2 p)=p+17$ are $p=7$, 31,$103 ;$ the only $p \equiv 23 \bmod 24$ for which $6 F(2 p)=p+1$ are $p=23,47$.

(4.2) Theorem. The only $p \equiv 11 \bmod 24$ such that $6 F(2 p)-4 F(p)=p-11$ is $p=11$; the only $p \equiv 19 \bmod 24$ such that $6 F(2 p)-4 F(p)=p+5$ are $p=19$, 43,67 .

(4.3) THEOREM. The only $p \equiv 1 \bmod 24$ such that $6 F(2 p)-6 F(p)=p-1$ are $p=73,97$; there is no $p \equiv 17 \bmod 24$ such that $6 F(2 p)-6 F(p)=p-17$.

(4.4) TheOREM. The only $p \equiv 5 \bmod 24$ such that $6 F(2 p)-6 F(p)=p-5$ is $p=5 ;$ the only $p \equiv 13 \bmod 24$ such that $6 F(2 p)-6 F(p)=p+11$ are $p=13,37$.

(4.7) Theorem. The only $p \equiv 3 \bmod 8$ such that $4 F(p)-2 F(2 p)=p-3$ are $p=3,11$.

(4.8) Theorem. The only $p \equiv 1 \bmod 8$ such that $6 F(p)-2 F(2 p)=p-1$ are $p=17,41 ;$ the only $p \equiv 5 \bmod 8$ such that $6 F(p)-2 F(2 p)=p+3$ are $p=5,29$.

The theorem (4.9) (obtained from (3.9)) is identical with (4.1).

(4.10) THEOREM. The only $p \equiv 3 \bmod 8$ such that $18 F(2 p)-4 F(p)=24 \omega(p)$ $+3 p-1$ is $p=3$; the only $p \equiv 11 \bmod 24$ such that $18 F(2 p)-4 F(p)=3 p-9$ is $p=11 ;$ the only $p \equiv 19 \bmod 24$ such that $18 F(2 p)-4 F(p)=3 p+39$ are $p=19$, 43,67 .

(4.11) THEOREM. The only $p \equiv 13 \bmod 24$ such that $6 F(2 p)-2 F(p)=p+19$ are $p=13,37$; the only $p \equiv 1 \bmod 24$ such that $6 F(2 p)-2 F(p)=p+15$ are $p=73,97 ;$ the only $p \equiv 17 \bmod 24$ such that $6 F(2 p)-2 F(p)=p-1$ is $p=17$; there is no $p \equiv 5 \bmod 24$ such that $6 F(2 p)-2 F(p)=p+3$.

(4.12) THEOREM. (4.1)-(4.11) contain all the singular relations with prime arguments implied by (1.2) with $s=4$.

\section{Proofs}

5. Proof of (2.1). This is practically obvious. However, if a formal proof be desired, a simple one is given by the identity

where

$$
2^{-s} \prod_{i=1}^{s}\left[1+\theta_{3}\left(q^{a_{i}}\right)\right]=\prod_{i=1}^{s}\left[1+\frac{1}{2}\left(\theta_{3}\left(q^{a_{i}}\right)-1\right)\right],
$$

$$
\theta_{3}(q)=\sum_{t=-\infty}^{\infty} q^{t^{2}}=1+2 \sum_{n=1}^{\infty} q^{n^{2}},|q|<1
$$

on the right of which the coefficient of $q^{n}$ is the $R$ function in (2.1). On the left the product is distributed before collecting the coefficient of $q^{n}$. 
6. Proof of (2.3). From the definitions of $R, R^{\prime}$, the left of (2.3) enumerates the same representations of $n$ as the right. For, on the left, all of $x^{2}, y^{2}, z^{2}, w^{2}$ may be unequal, or two may be equal and distinct from the remaining two which may be either equal or unequal, or three may be equal and distinct from the fourth, or all four may be equal, and these cases are exhaustive and mutually exclusive. To account for the numerical factors $1,6,3,4,1$, consider one, say 6 . We have

$$
R^{\prime}\left(n=x^{2}+y^{2}+2 z^{2}\right)=R^{\prime}\left(n=x^{2}+y^{2}+z^{2}+z^{2}\right) .
$$

From a particular representation $x^{2}, y^{2}, z^{2}, z^{2}$, since $x^{2}, y^{2}, z^{2}$ are unequal, there are only 2 representations, obtained by permuting $x^{2}, y^{2}$ contributed to the left of (2.3). But on the left of (2.3) the equal squares (if any) in the representations enumerated are free in position. If in the particular representation the $z^{2}$ s are free, the contribution to the left is $4 ! /(1 ! 1 ! 2 !),=12$. Hence $R^{\prime}\left(n=x^{2}+y^{2}+2 z^{2}\right)$ must be multiplied by $12 / 2,=6$.

All proofs are now completed.

Californta Institute of Technology, Pasadena, Calif. 